\title{
Bilgisayar Destekli DC Motor Hız Denetimi
}

\author{
Seda POSTALCIOĞLU ${ }^{1 *}$ (D) , Çetin KÖKTÜRK ${ }^{2}$ \\ Bolu Abant İzzet Baysal Üniversitesi, Mühendislik Fakültesi, Bilgisayar Mühendisliği Bölümü \\ Bolu Abant İzzet Baysal Üniversitesi, Mühendislik Fakültesi, Elektrik Elektronik Mühendisliği Bölümü²
}

Geliş / Received: 08/01/2019, Kabul / Accepted: 13/05/2019

Öz

DC motorlar günümüzde birçok alanda kullanılmaktadır. Bu çalışmada DC motor hızının Matlab ile denetlenmesi amaçlanmıştır. Sistemin tasarımında Arduino Uno mikrodenetleyici kartı kullanılmıştır. Arduino

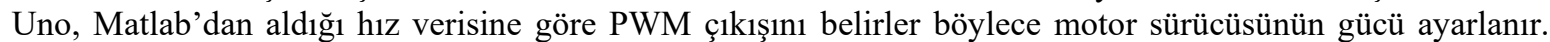
Denetleyici olarak PID kullanılmıştır. Sonuçlardan görüleceği üzere motor hızı PID ile bilgisayar destekli olarak kontrol edilmiştir. Gerçekleştirilen bu çalışma kontrol eğitimi açısından da verimli olacağı düşünülmektedir.

Anahtar Kelimeler: PID denetim, DC motor, seri haberleşme

\section{Computer Aided DC Motor Speed Control}

\begin{abstract}
DC motors are used in many areas today. In this study, it is aimed to control the DC motor speed with Matlab. Arduino Uno microcontroller card is used in the design of the system. The Arduino Uno determines the PWM output according to the speed data it receives from Matlab, so the output of the motor driver is set. PID is used as controller. It can be seen from the results, the motor speed is controlled by PID with Matlab. This study could be efficient in terms of control education.
\end{abstract}

Keywords: PID control, DC motor, serial communication

\section{Giriş}

Bir DC motorun gerçek zamanlı hız denetimi birçok alanda istenen bir fonksiyondur. Günlük hayatta karşımıza çıktığı gibi endüstride yüksek güç gerektiren birçok alanda kullanılmaktadır. DC motorlar güç elektroniği devreleri ile kontrol edilebilir. Fakat böyle bir sistemin üzerinde değişiklik yapılması kolay değildir. $\mathrm{Bu}$ tarz sistemler yazılım ile birleştirildiği zaman çok daha esnek olabilmektedirler. Örneğin, kimya alanında kullanılan karıştırmalı tank reaktör (Continuous Stirred - Tank Reactor) sisteminde farklı zamanlarda, farklı hızlarda karıştırma yapmasını arzu ettiğimizde bunu bir bilgisayar yardımıyla yapmak çok daha kolay olacaktır. DC motorların PID ile hız denetimleri hakkında yapılmış birçok çalışma bulunmaktadır. 2001 yılında yapılan bir çalışmada, Matlab kullanılarak elde edilen katsayılar bir sayısal işaret denetleyicisinin assembly kodlarına yerleştirilerek, PID denetimi gerçek zamanlı olarak sağlanmıştır (Tang ve Chassaing, 2001). 2012 de yapilan bir çalışmada Bulanık-PID kontrol firçasız motorlar için incelenmiştir (Fan ve Zhu $\mathrm{H}, 2012)$. DC motor kullanılarak bulanık mantık ve PID kontrol çalışılmıştır (Gubara vd.,2016; Almatheel ve Abdelrahman 2017). PID denetleyiciye dayalı olarak, dc motor hız kontrolünde optimizasyon teknikleri üzerine 
bir çalışma yapılmıştır (Suman and Giri , 2016). Yine dc motor üzerine yapılmış farklı çalışmalar Viola vd. (2017), Postalcıoğlu ve Alişiroğlu (2018)' de görülmektedir.

\section{Materyal ve Metot}

Çalışmada seri haberleşme ile dc motor hız denetimi PID denetleyici kullanılarak Matlab ortamında gerçekleştirilmiştir. Sistemin temel yapısı Şekil 1'de verilmiştir.

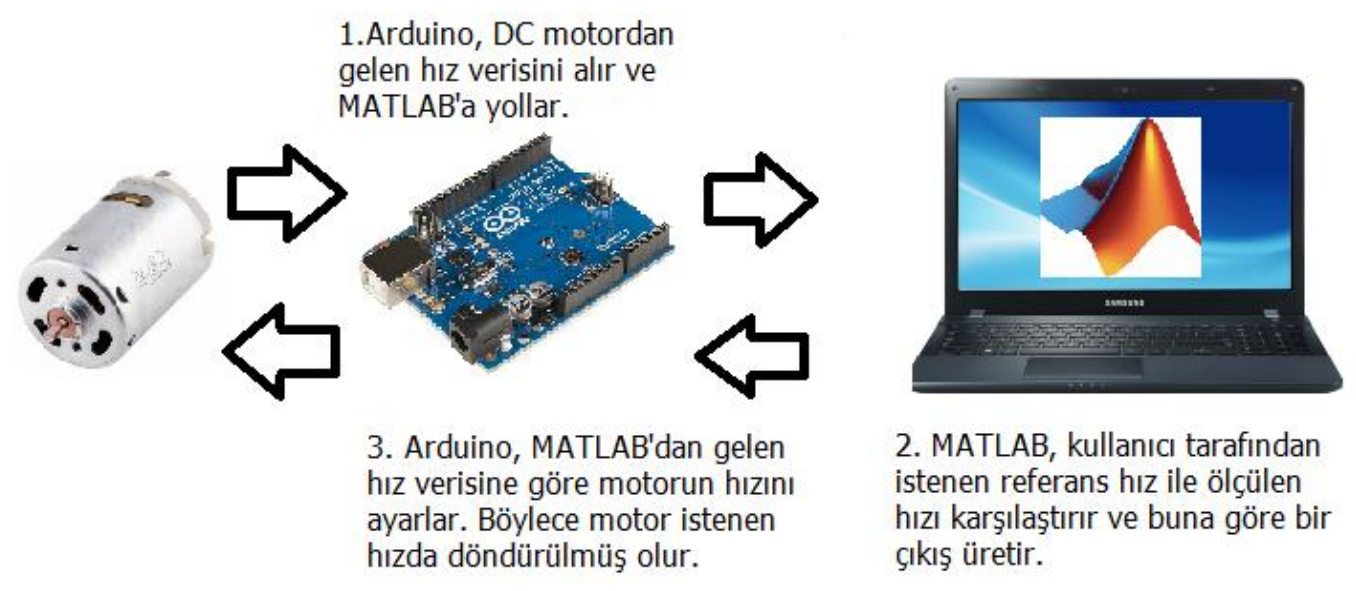

Şekil 1. Sistemin temel yapısı

Sistemin arayüzü Matlab-Gui ile gönderir. Matlab, bu yeni hız ile istenen hazırlanmıştır ve Şekil 2'de verilmiştir. referans hızı karşılaştırır ve hatayı hesaplar. Arayüze $\mathrm{Kp}, \mathrm{Ki}, \mathrm{Kd}$ değerleri ve motorun yerleşmesi istenen hiz girilir. Matlab bu katsayıları alır ve PID denklemine yerleştirir. Daha sonra ürettiği kontrol sinyalini Arduino'ya, Arduino da motor sürücüye gönderir. Motor kontrol sinyaline göre bir hızda dönmeye başladıktan 1 saniye sonra Arduino motorun yeni hızını okur ve Matlab'a

Eğer arka arkaya iki hata değeri sıfır ölçülmüşse, sistem istenen hıza yerleşmiş demektir. Program ölçtüğü hı verilerini grafiğe döker ve çalışmayı durdurur. Katsayılar panelinden uygun parametre değerleri $\mathrm{Kp}, \mathrm{Ki}, \mathrm{Kd}$ atanır. 


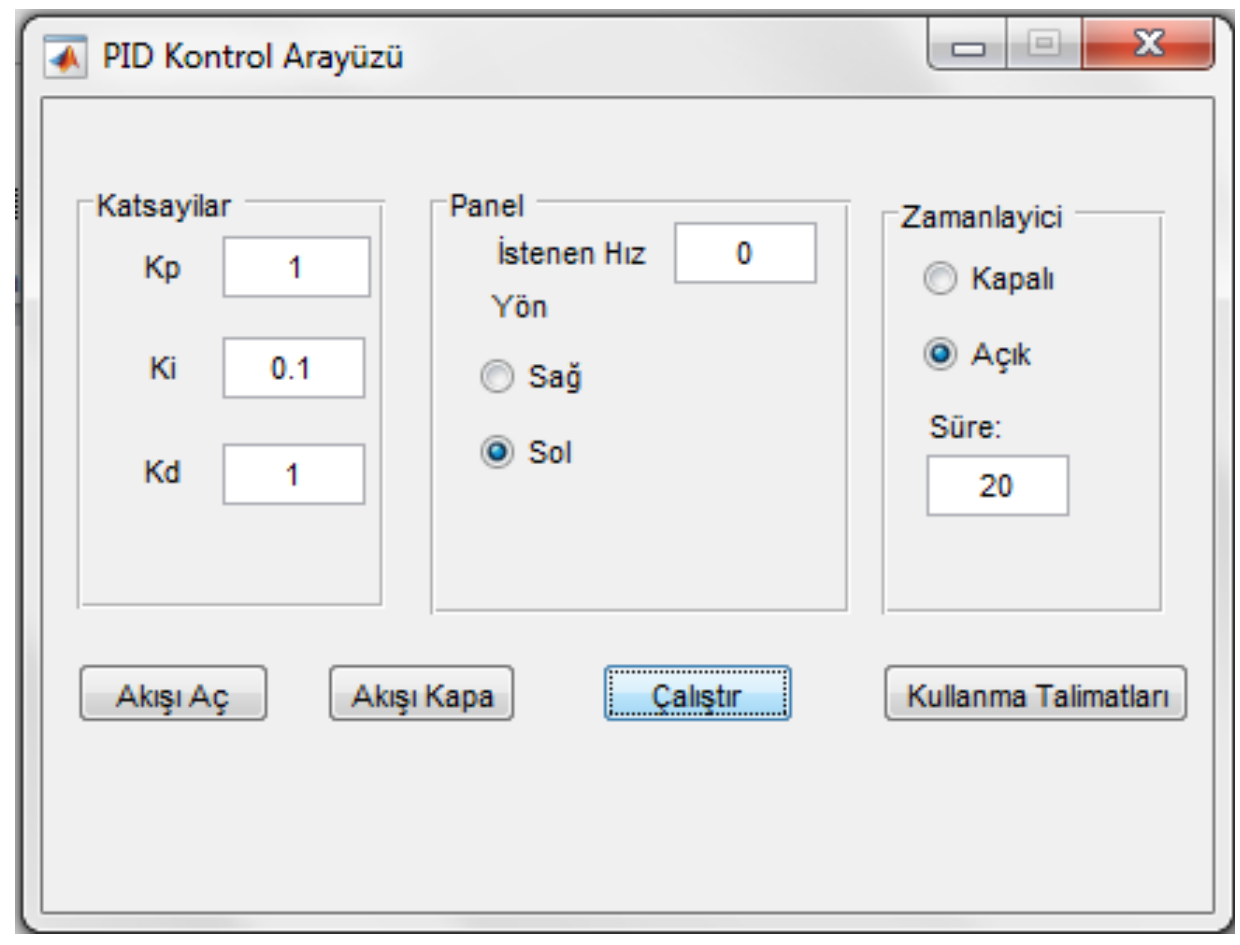

Şekil 2. PID denetimi için kullanıcı arayüzü

Ortada bulunan panel k1sminda ise, sistemimizin yerleşmesi istenen hız seçilir. Sağ kısımda bulunan panelde ise, zamanlayıcı modülü istenirse açılıp kapanabilir. Zamanlayıcı modülü ile sistemin çalışması için istenen süre saniye cinsinden girilir. $\mathrm{Bu}$ süre içerisinde eğer sistem istenen hıza yerleşirse program çalışmayı durdurur ve hız zaman grafiğini çizer. Eğer yerleşmez ve zaman dolarsa, yine aynı şekilde program çalışmayı durdurur ve grafiği çizer, daha sonra kontrolü tekrar kullanıcıya verir.

Motor çalıştırılmadan önce seri haberleşme veri akışının açılması gerekmektedir. Bunun için Akışı Aç tuşuna basılır. Eğer Arduino Uno'nun bilgisayar ile fiziksel bağlantıs1 kesilecek veya Arduino Uno baştan başlatılacak olursa, önceden Akışı Kapa butonuna basılarak bağlantının güvenli bir biçimde kapatılması gerekmektedir. Aksi takdirde arayüzün kapatılıp tekrar açılması gereklidir. Bütün parametreler ayarlandıktan sonra Çalıştır butonuna basılarak sistem çalıştırılır. Kullanma Talimatları butonuna basarak da sistemin çalıșmasına ait talimatlar k1saca görülebilir.

\section{Bulgular}

Şekil 3'de Bilgisayar destekli dc motor hiz denetimi uygulaması görülmektedir. Arduino Uno, bilgisayar ile veri alışverişi yapmaktadır. Arduino-Uno, bilgisayarda üretilen kontrol sinyalini seri haberleşme ile alır. Daha sonra bu sinyali, motoru süren gürültülü devre ile Arduino'nun bulunduğu devreyi elektriksel olarak izole eden optokuplörlere yollar. Optokuplörler de bu sinyali motor sürücünün hız ve yön denetimini yapan uçlarına iletir. Motor yeni hızında dönmeye başlar, sensör de motorun her çeyrek dönüşünde Arduino Uno'daki sayaç değişkenini 1 arttırır. Her saniye bilgisayardan yeni veri geldiğinde ise sayaçtaki sayı bilgisayara iletilir ve sıfırlanır. Uygulama devre şeması şekil 4'de verilmiştir. 


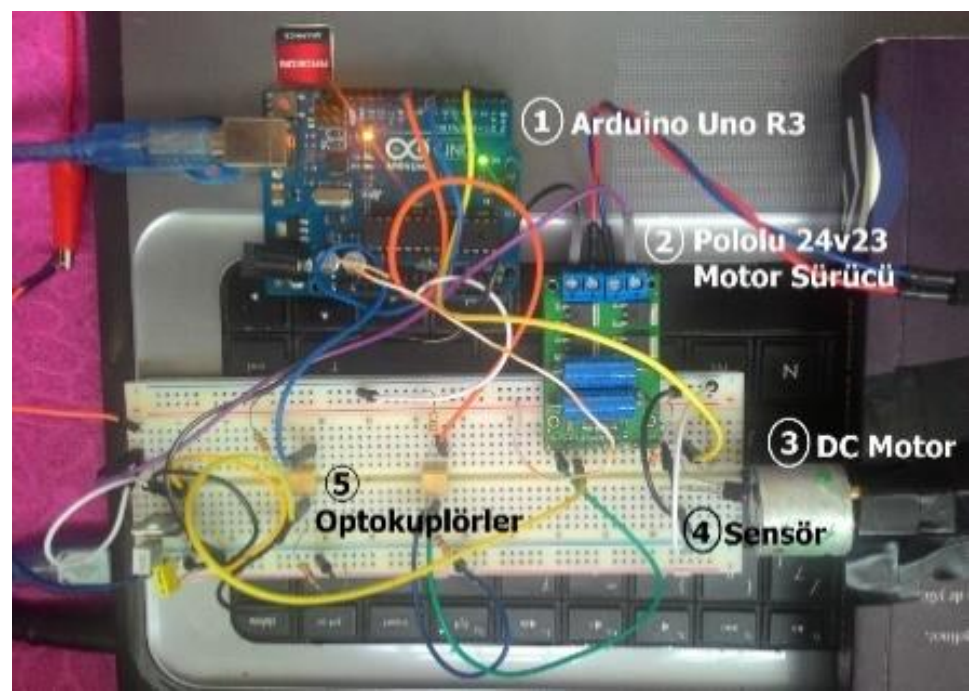

Şekil 3. Bilgisayar destekli dc motor hız denetimi uygulaması

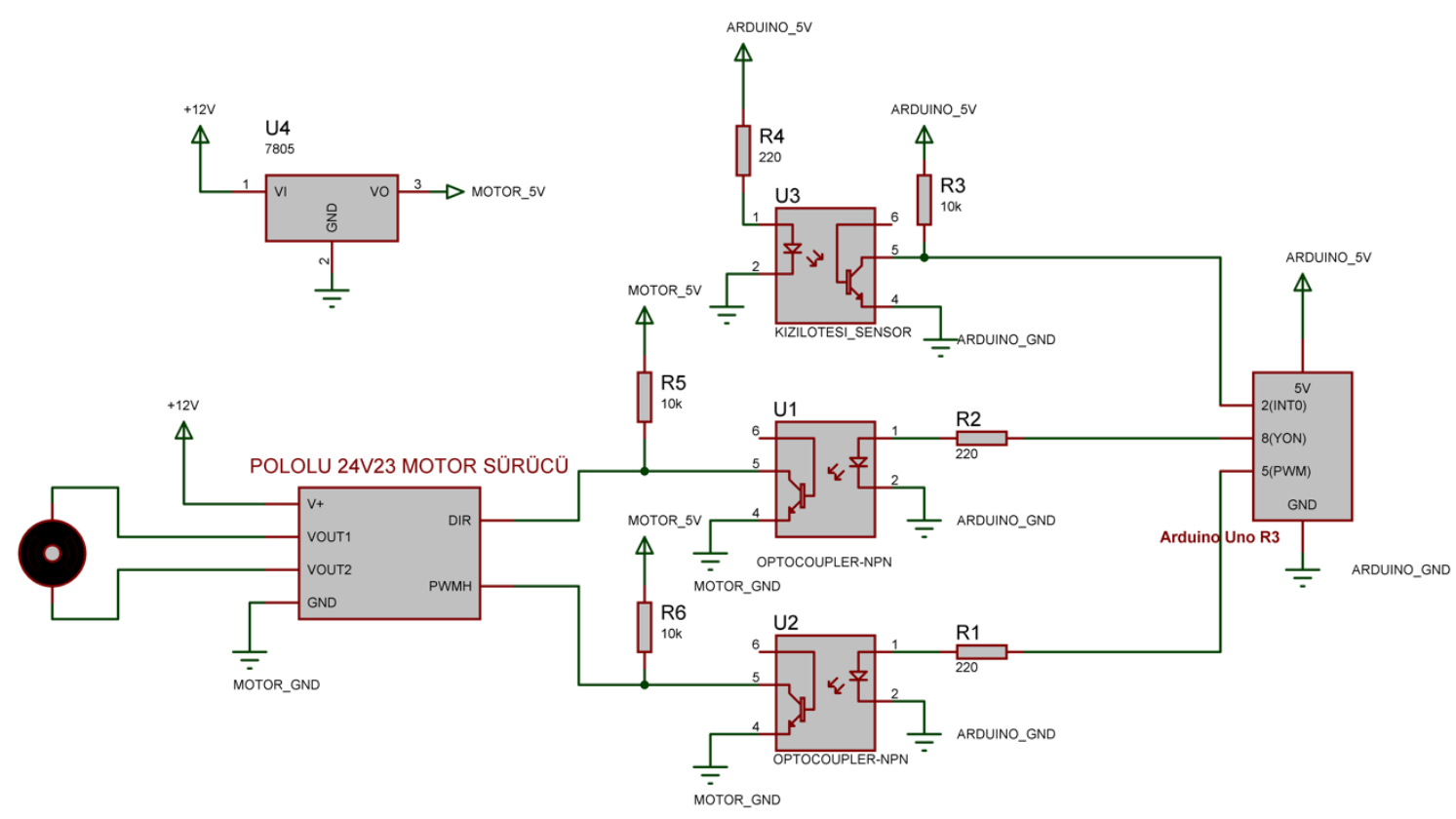

Şekil 4. Seri haberleşme ile dc motor hız denetimi için devre şeması

PID denetim için $K_{p}, K_{i}$ ve $K_{d}$ değerleri şekil eğitimi açısından da bu tarz bir sistemin 2'de gösterilen arayüzden girilmiştir. kullanımı oldukça faydalı olacaktır.

Sonuçlar şekil 5'de verilmiştir. Sonuçlardan görüldüğü üzere, P, PI, PID denetiminin sistemin cevabına etkileri gözlenmektedir. DC motorun bilgisayar destekli kontrolü insan hatalarını ortadan kaldıracağı gibi kontrol 


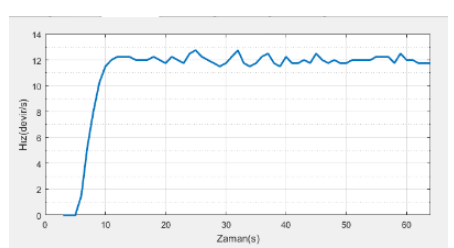

a) P Denetim

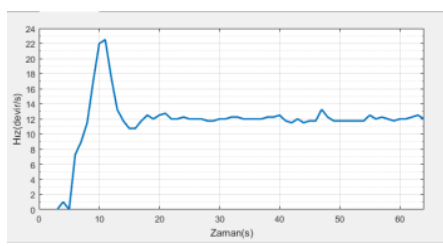

b) PI Denetim

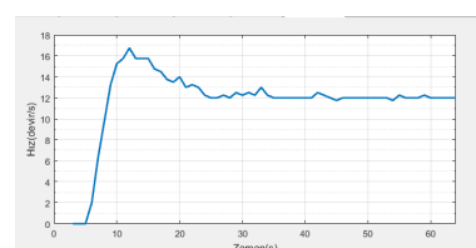

c) PID Denetim

Şekil 5. DC motor için P,PI,PID denetim sonuçları

\section{Sonuç}

Çalışmada bilgisayar destekli DC motor hızının denetlenmesi amaçlanmıştır. Tasarlanan sistemde Arduino Uno mikrodenetleyici kartı ve Matlab kullanılmıştır. Denetleyici olarak PID kullanılmıştır. Sonuçlardan görüleceği üzere motor hızı PID ile bilgisayar destekli olarak kontrol edilmiştir. Gerçekleştirilen bu çalışma ile kontrol eğitimi açısından da uygulamalı anlatım için verimli olacağı düşünülmektedir.

Sonraki çalışmalar için ise sisteme arıza tespit modülü eklenmesi planlanmaktadır. Aşırı 1sınma, aşırı akım, sensör arızaları vb. durumlar oluştuğunda arayüz üzerinden kullanıcı ile haberleşerek sistem daha güvenli hale getirilebilir. Ayrica sistem, internet üzerinden haberleştirilerek de kullanıma sunulabilir.

\section{Teşekkür}

Gerçekleştirilen çalışma Bolu Abant İzzet Baysal Üniversitesi, Öğrenci Bilimsel Araştırma Projesi olarak 2016.09.03.1008 proje numarası ile desteklenmiştir.

\section{Kaynaklar}

Almatheel Y.A.,Abdelrahman A. 2017. "Speed control of DC motor using Fuzzy Logic Controller," 2017 International Conference on Communication, Control, Computing and Electronics Engineering
ICCCCEE), pp. 1-8. doi: 10.1109/ICCCCEE.2017.7867673

Fan S.,Zhu H,. 2012."Simulation of the fuzzy PID control system for Brushless DC motors based on MATLAB," International Conference on Automatic Control and Artificial Intelligence (ACAI 2012),pp. 18541857.doi: 10.1049/cp.2012.1353

Gubara W, Elnaim M, Babiker S.F. 2016. "Comparative study on the speed of DC motor using PID and FLC," 2016 Conference of Basic Sciences and Engineering Studies (SGCAC),pp.24-29.

doi:10.1109/SGCAC.2016.7458001.

Postalcıoğlu, S., Alişiroğlu, Y. 2018. "Real Time DC Motor Speed/Position Control with Bluetooth Communication". International Journal Of Engineering and Computer Science, 7(02), 23663-23668, 2018.

Suman S.K.,Giri V.K. 2016. "Speed control of DC motor using optimization techniques based PID Controller," 2016 IEEE International Conference on Engineering and Technology (ICETECH),pp. 581587.doi:10.1109/ICETECH.2016.7569318.

Tang, J., Chassaing, R. 2001."PID controller using the TMS320C31 DSK with online parameter adjustment for real-time DC motor speed and position control". Industrial 
Electronics, 2001. Proceedings. ISIE 2001.

IEEE International Symposium , 786 -791.

Viola J.,Angel L.,Sebastian J.M. 2017.

"Design and robust performance evaluation of a fractional order PID controller applied to a DC motor," in IEEE/CAA Journal of Automatica Sinica, vol. 4, no. 2, pp. 304-314. doi: 10.1109/JAS.2017.7510535 